Théologiques

Théologiques

\title{
Néantisation et relationalité chez NISHIDA Kitarô et WATSUJI Tetsurô
}

\section{Jacinthe Tremblay}

Volume 4, numéro 2, octobre 1996

L'épreuve du rien

URI : https://id.erudit.org/iderudit/602440ar

DOI : https://doi.org/10.7202/602440ar

Aller au sommaire du numéro

\section{Éditeur(s)}

Faculté de théologie de l'Université de Montréal

\section{ISSN}

1188-7109 (imprimé)

1492-1413 (numérique)

Découvrir la revue

Citer cet article

Tremblay, J. (1996). Néantisation et relationalité chez NISHIDA Kitarô et WATSUJI Tetsurô. Théologiques, 4(2), 63-82. https://doi.org/10.7202/602440ar
Résumé de l'article

Le néant absolu (zettai $\mathrm{mu}$ ) a suscité divers types de discours dans la philosophie japonaise contemporaine, notamment celui de la relationalité, dont traitent principalement NISHIDA Kitarô (1870-1945) et WATSUJI Tetsurô (1889-1960). Nishida met en lumière le lien intrinsèque entre le néant absolu et la relationalité en montrant que l'individuel (kobutsu) est tel uniquement par le fait de se confronter à un autre individuel, et que cette confrontation est un processus de néantisation absolue par lequel l'individuel renvoie constamment à l'autre, ce qui permet en retour une auto-affirmation absolue. Le néant absolu est également le fondement de la structure relationnelle de Watsuji, qui fait l'originalité de son éthique. L'humain (ningen), à la fois individuel et social, comporte une négation par laquelle il nie l'ensemble de l'humanité, se constituant ainsi comme individu et comme société. Tant la philosophie de Nishida que celle de Watsuji convergent vers le néant absolu et s'articulent autour de lui.
Tous droits réservés @ Faculté de théologie de l'Université de Montréal, 1996

Ce document est protégé par la loi sur le droit d'auteur. L'utilisation des services d'Érudit (y compris la reproduction) est assujettie à sa politique d'utilisation que vous pouvez consulter en ligne.

https://apropos.erudit.org/fr/usagers/politique-dutilisation/ 


\title{
Néantisation et relationalité chez Nishida Kitarô et Watsujı Tetsurô ${ }^{1}$
}

\author{
Jacinthe TREMBLAY
}

\section{RÉSUMÉ}

Le néant absolu (zettai mu) a suscité divers types de discours dans la philosophie japonaise contemporaine, notamment celui de la relationalité, dont traitent principalement NiSHIDA Kitarô (1870-1945) et WATSUJI Tetsurô (1889-1960). Nishida met en lumière le lien intrinsèque entre le néant absolu et la relationalité en montrant que l'individuel (kobutsu) est tel uniquement par le fait de se confronter à un autre individuel, et que cette confrontation est un processus de néantisation absolue par lequel l'individuel renvoie constamment à l'autre, ce qui permet en retour une auto-affirmation absolue. Le néant absolu est également le fondement de la structure relationnelle de Watsuji, qui fait l'originalité de son éthique. L'humain (ningen), à la fois individuel et social, comporte une négation par laquelle il nie l'ensemble de l'humanité, se constituant ainsi comme individu et comme société. Tant la philosophie de Nishida que celle de Watsuji convergent vers le néant absolu et s'articulent autour de lui.

Absolute nothingness (zettai mu) has provoked diverse discourses in contemporary Japanese philosophy, notably that of relationality, treated principally by NISHIDA Kitarô (1870-1945) and WATSUJI Tetsurô (1889-1960). Nishida illuminates the intrinsic link between absolute nothingness and relationality by showing that the individual (kobutsu) is such as she or he is, uniquely by virtue of confronting another individual. This confrontation is a process of absolute negation by which the individual constantly refers to the other, which in return, permits an absolute self-affirmation. Absolute nothingness is equally the foundation of the relational structure of Watsuji which is what makes his ethics original. The human being (ningen), at once individual and social comprises a negation by which he denies the whole of humanity thus constituting himself as individual and as society. Both the philosophies of Nishida and Watsuji converge towards absolute nothingness, and both hinge on this concept.

1 Cet article a été écrit grâce à une subvention du C.R.S.H. Je remercie le professeur Bernard Bernier, du Centre d'études de l'Asie de l'est de l'Université de Montréal, pour ses suggestions et commentaires. 
Exception faite de NISHTANI Keiji ${ }^{2}$, dont la préoccupation principale a été de mettre en lumière le sunyata ${ }^{3}$, le néant absolu (zettai $m u$ ) n'est pas comme tel un thème de discours dans la philosophie japonaise contemporaine. Il n'en a pourtant pas moins une importance déterminante puiqu'il a suscité, en tant que fondement ultime de la réalité, divers types de discours, principalement la logique du basho (lieu) de NISHIDA Kitarô, la logique de l'espèce et la métanoétique de TANABE Hajime ${ }^{4}$, et l'éthique de WATSUjI Tetsurô. Le discours qui a suscité le plus d'intérêt jusqu'ici, tant au Japon qu'en Occident, est le discours religieux. L'exemple le plus éminent de cela est l'École de Kyôto ${ }^{5}$ qui, s'inspirant des essais de philosophie de la religion de Nishida, s'est arrêtée surtout aux rapports entre le néant absolu du bouddhisme zen et le Dieu chrétien ${ }^{6}$. Il $\mathrm{y}$ a toutefois un autre thème de la philosophie japonaise, intrinsèquement relié à la question du néant absolu, qui n'a été que très peu considéré jusqu'à maintenant : la relationalité. Ce thème se trouve principalement chez Nishida et chez Watsuji.

Nishida apporte une contribution fort originale à la question de la néantisation et de la relationalité lorsqu'il affirme que l'individuel (kobutsu) est l'individuel par le fait de se confronter (taisuru) à l'individuel. La question à laquelle vient répondre sa logique paradoxale, qui se fonde dans le néant absolu, est celle d'un individuel totalement indépendant et auto-déterminé qui pourtant, n'est individuel que dans la mesure où il se confronte à un autre individuel. Cette confrontation n'est autre qu'un processus de néantisation absolue par lequel l'individuel renvoie constamment à l'autre, ce qui permet en retour son affirmation absolue.

Le néant absolu est également le fondement de l'éthique de Watsuji. La logique paradoxale de Nishida et l'éthique de Watsuji se fondent dans une structure relationnelle semblable. Cette dernière repose en effet sur les relations fondamentales établies entre l'humain et l'humain, l'humain

2 Voir le «Glossaire " à la fin de l'article.

3 Le sunyata renvoie à la non-substantialité de toute chose. C'est la version religieuse du néant absolu.

4 Voir le "Glossaire ".

5 Voir le « Glossaire ".

6 Voir à ce propos Robert A. CARTER, The Nothingness Beyond God: An Introduction to the Philosophy of Nishida Kitarô. New York, Paragon House, 1989, 191 p.; Jacynthe TREMBLAY, “ La logique de Nishida ", Sciences religieuses 22/3 (1993) 337-349; Hans W ALDENFELS, Absolute Nothingness: Foundations of a Buddhist-Christian Dialogue. New York, Paulist Press, 1980, 214 p.; YUSA Michiko, "The Religious Worldview of Nishida Kitarô'», The Eastem Buddhist 20/2 (1989) 63-76. 
et la famille, l'humain et la société. L'individu (kojin) n'y est jamais séparé de la communauté et de la société puisqu'il peut être compris uniquement à partir de la totalité des relations humaines. Une des notions déterminantes de l'éthique de Watsuji est l'aidagara ou relationalité qui réside dans les diverses relations entre humains (ningen). Etre humain signifie exister sous le mode de la relationalité. Cette philosophie permet de bien saisir la pensée et le comportement japonais, basés sur la manière de vivre dans un réseau de relations.

\section{Le néant absolu}

Deux postulats sont centraux dans la philosophie occidentale et ce, depuis les débuts de la philosophie grecque. Le premier est qu'il existe, derrière la multiplicité, une réalité-substance stable. Selon les époques et les penseurs, cette réalité-substance stable a été interprétée comme atome, eau, air ou matière indéfinie. Le second postulat est qu'elle ne peut être saisie que par l'esprit bien qu'elle soit approchée par et dans les organes des sens. Partant, la philosophie est une activité purement cognitive, et l'expérience ne sert qu'à fournir de l'information qui est restructurée par la raison.

Il est courant, dans la tradition du bouddhisme zen, de dire que le zen est anti-philosophique, et donc anti-rationnel, anti-discursif et anticonceptuel, s'il est vrai que les notions de rationalité, de discursivité et de conceptualisation sont comprises dans le mot "philosophie". De plus, et c'est là la spécification de l'ensemble des pensées et philosophies orientales ${ }^{7}$, la réalité ultime n'est pas l'être ou une substance stable mais le néant absolu ou un indéterminé qu'on retrace derrière les concepts, le langage et la logique habituelle. De plus, le donné fondamental du zen est l'expérience. C'est la raison pour laquelle les concepts et le langage manquent le but du zen. Dans la mesure où la réalité que vise le zen est

7 Les termes " Orient " et "Occident ", que nous utilisons pour des raisons de commodité, n'en sont pas moins imprécis. L'Orient comporte plusieurs cultures qui sont passablement différentes, nonobstant certains traits similaires. La culture japonaise, par exemple, n'a que très peu de choses semblables à la culture indienne et demeure fort différente de la culture chinoise (dont elle provient pourtant en bonne partie). La dichotomie Orient-Occident est d'autant plus inadéquate qu'on retrace souvent des lignes de pensée énonnamment proches dans des traditions culturelles fort différentes, par exemple, dans le bouddhisme zen et chez Eckhart. En effet, des questions similaires ont été discutées dans des cultures différentes, quoique en des concepts souvent non semblabes. Voir NAKamURA Hajime, "Methods and Significance of Comparative Philosophy ", Revue Internationale de Philosophie 28/107. 110 (1974) 184-193. 
pré-linguistique et précède la séparation sujet-objet, elle est le fondement de tout; elle est le sans-forme et le formé. Il faut préciser ici que le formé, c'est-à-dire toutes les choses qui existent dans ce monde, n'a pas d'essence déterminée à la manière aristotélicienne. En fait, "there are absolutely no fixed essences behind the ever-changing forms of phenomena ${ }^{8}$ ». Ce n'est pas une fluidité ontologique qu'on trouve derrière les choses mais le sans-forme, le néant absolu indifférencié, qui ne peut être indiqué à l'aide du langage et des concepts mais uniquement par le silence. Les mots, en effet, peuvent indiquer seulement les différences entre les choses et non pas leur source ultime. Il faut donc mettre l'accent à la fois sur la forme extérieure et sur le fondement, sur l'individuel et son basho (lieu) ${ }^{9}$, c'està-dire le néant absolu.

Avoir le sens du fondement est, en même temps, avoir le sens de la fluidité des choses différenciées. Nous croyons habituellement que les choses elles-mêmes sont le centre de notre perception, et que la distinction sujet-objet est la réalité unique et finale. Pourtant, les catégories dualistes ne sont pas applicables à l'illumination zen, qu'il s'agisse du sujet et de l'objet, de l'âme et du corps, de l'être et du non-être, ou encore, de ce qui connaît et de ce qui est connu. C'est d'ailleurs là ce que SUZUKI Daisetsu Teitarô s'est efforcé de mettre en lumière tout au long de sa longue carrière ${ }^{10}$. La connaissance donnée par le zen ne fait pas de distinction entre ces couples d'opposés et les saisit en une seule et même pensée. De là résulte l'illumination dans laquelle il n'y ni dualité ni dis-

8 Robert E. CARTER, «Toward a Philosophy of Zen Buddhism. Prolegomena to an Understanding of Zen Experience and Nishida's Logic of Place », Eastern Buddhist 13/2 (1980) 127-130; p. 128.

9 Le mot japonais "basho", qui s'inspire de la chôra platonicienne, du topos aristotélicien et du Feld de la physique et du néo-kantisme, signifie à l'origine " champ », « lieu », " topos ». Sous l'influence de Nishida, qui en fait un terme majeur de sa philosophie, le mot "basho" est devenu un mot technique de la philosophie contemporaine. Nishida donne au basho plusieurs acceptions. C'est d'abord l'universel en quoi se situe le sujet grammatical, puis la conscience où advient l'intellection et où le soi se voit lui-même. Nishida considère que les catégories les plus hautes, qui sont le vrai, le beau et le bien, peuvent être ramenées au cogito, lequel constitue la conscience en général transcendantale, le prédicat des prédicats. Il fonde donc le rôle des prédicats sur la catégorie, comprise comme concept de haut degré, et pose la conscience comme le plus haut prédicat du jugement. Dans ces conditions, le cogito est l'universel le plus élevé avant que ne soit finalement atteint le basho du néant absolu (zettai mu no basho), qui est l'universel le plus englobant. Ajoutons que le terme " basho " a l'avantage de ne comporter aucune connotation psychologique et de tout englober. Watsuji reprendra ce terme de Nishida. 
crimination. Dans l'illumination, l'être intérieur

est purifié et se voit tel qu'il est réellement, c'est-à-dire non comme un ego s'affirmant en contraste avec le non-ego, mais comme quelque chose qui dépasse les opposés et cependant les synthétise en soi. Ce qui est détruit, c'est le dualisme des choses et non leur unité. Et libération signifie retour à sa demeure d'origine. Par conséquent, la vision intérieure consiste à voir l'unité dans la multiplicité, à comprendre l'opposition de deux idées non pas comme une dépendance réciproque, mais comme un couple jaillissant d'un principe plus haut ${ }^{11}$.

Le dualisme s'évanouit de lui-même lorsque l'esprit demeure paisiblement dans l'unité des choses. Le fondement de la philosophie du zen réside donc dans l'unité absolue des choses plutôt que dans les dualismes. Toute chose déterminée est une articulation du sans-forme ou du néant absolu. Il faut voir les choses individuelles dans leur indétermination pour saisir l'inarticulé qui se situe derrière la conceptualisation ou la différenciation. Ainsi, " a 'particular thing' reveal itself to us in its original reality. This is Prajna wisdom, or non-discriminating consciousness ${ }^{12}$ ".

La métaphysique occidentale a pour sa part toujours prôné la priorité ontologique de l'être $(y \hat{u})$ sur le non-être ( $m u$, néant relatif). Dans son article intitulé "Non-Being and $M u$. The Metaphysical Nature of Negativity in the East and the West ", ABE Masao remet cette évidence en question de manière radicale lorsqu'il mentionne qu'il ne voit pas le fondement ontologique sur lequel s'appuie l'affirmation voulant que l'être ait la priorité sur le non-être ${ }^{13}$. Dans la même perspective, il faut également demander ce qui justifie le fait qu'on accorde priorité à la vie sur la mort et au bien sur le mal ${ }^{14}$. Selon Abe, le fondement qui embrasse à la fois l'être $(y \hat{u})$ et le non-être $(m u)$ est le néant absolu (Zettai $m u)$. Loin de s'opposer à l'être comme le fait le non-être, il permet plutôt de montrer l'articulation entre les deux.

L'émergence du bouddhisme dans l'Inde ancienne a favorisé la mise en lumière de l'importance de la négativité. Un des principes fondamentaux du bouddhisme est que chaque chose est dépendante d'une autre

11 SUZUKI Daisetsu Teitarô, Essais sur le bouddhisme zen, Première série. [Spiritualités vivantes, 9], Paris, Albin Michel, 1972, 471 p.; p. 170.

12 Robert E. CARTER, " Toward a Philosophy of Zen Buddhism ", p. 128.

13 Voir ABE Masao, "Non-Being and $\mathrm{Mu}$. The Metaphysical Nature of Negativity in the East and the West ", Religious Studies 11/2 (1975) 181-192; p. 181.

14 A BE Masao, “ Non-Being and Mu ", p. 183. 
chose, autrement dit, qu'il n'y a rien d'indépendant et d'auto-suffisant. Ce principe, appelé pratitya-samutpada, signifie co-surgissement dépendant, relationalité; il est inséparable de deux autres principes bouddhistes fondamentaux, à savoir l'absence de moi éternel (anatman) et l'absence de substance immuable.

Le philosophe indien Nagarjuna (vers le $\mathrm{II}^{\mathrm{e}}$ ou III ${ }^{\mathrm{e}}$ siècle) ${ }^{15}$ a montré les implications de ces principes bouddhistes fondamentaux en élaborant l'idée de sunyata ( $k u$, vide, ou zettai $m u$, néant absolu). Le néant absolu n'est pas l'exact opposé de l'être puisque ce serait là accorder de nouveau la priorité à un principe sur un autre, en l'occurrence à la négativité sur la positivité. Ce n'est pas, non plus, le point médian entre les deux extrêmes que sont l'être et le non-être. C'est plutôt une voie (la "voie du milieu» de Nagarjuna) qui transcende et embrasse à la fois toute dualité possible, incluant celles de l'être et du non-être, de l'affirmation et de la négation, de la vie et de la mort, du bien et du mal. Elle n'est pas basée simplement sur une négation, mais sur une négation de la négation, sur une négation absolue qui n'est rien d'autre qu'une affirmation absolue. La remise en question de la priorité du principe positif sur le principe négatif implique que les deux ont une force égale et se nient mutuellement. Leur fondation dans le néant absolu permet de dépasser leur caractère dualiste et les rend entièrement relatifs, complémentaires et réciproques.

\section{2 . NiSHIDA Kitarô. Les rappports inter-individuels ${ }^{16}$}

Nishida s'est efforcé, tout au long de sa carrière, de mettre en lumière l'unité du sujet et de l'objet plutôt que leur séparation. Il n'est parvenu que tardivement (1927) à montrer que le basho du néant absolu (zettai mu no basho) est le fondement de l'unification subjectivo-objective, le lieu d'où surgissent et vers lequel convergent les termes contradictoires. Nishida a d'abord identifié la condition préalable à la distinction sujet-objet en tant qu'expérience pure :

L'expérience pure signifie connaître la réalité telle qu'elle est. C'est par conséquent connaître conformément à la réalité en renonçant entièrement aux artifices du soi. Puisque ceux qui discutent habituel-

Nagarjuna a établi l'École Mâdhyamika et est considéré, en Chine et au Japon, comme le fondateur du bouddhisme mahayana. À propos de la philosophie de Nagarjuna, voir par exemple : ABE Masao, «Zen and Western Thought ", International Philosophical Quarterly 10/4 (1970) 501-541; Kenneth K. INADA, "Nâgârjuna and Beyond ", Journal of Buddhist Philosophy 2 (1984) 65-76; YAMAUCHI Tokuryû, "Problems of Logic in Philosophy East and West », Japanese Religions 3/3 (1963) 1-7.

16 Voir le « Glossaire ». 
lement de l'expérience y joignent en fait toute sorte d'idées, "pure " signifie précisément la condition de l'expérience en elle-même, en dehors de l'ajout de toute pensée ou discrimination. Par exemple, elle signifie qu'à l'instant où on voit une couleur ou qu'on entend un son, l'expérience précède non seulement la pensée (qu'il s'agisse de la fonction d'une chose extérieure ou de ma sensation), mais qu'elle précède même l'ajout du jugement consistant à savoir de quelle couleur ou de quel son il s'agit. Donc, l'expérience pure est identique à l'expérience immédiate. Il n'y a encore ni sujet ni objet lorsqu'on fait immédiatement l'expérience d'un stade conscient de la conscience; le connaître et l'objet sont entièrement un. C'est la plus pure forme d'expérience ${ }^{17}$.

L'intuition fondamentale qu'avait Nishida en élaborant sa notion d'expérience pure est que les éléments contradictoires qu'on trouve dans la vie humaine, qu'il s'agisse de l'esprit et de la matière, de l'individuel et du tout, de l'individu et du monde, du sujet et de l'objet, ne s'excluent pas l'un l'autre mais sont fondamentalement en harmonie. Ils sont secondaires par rapport à l'expérience pure et se fondent sur elle. Il n'y a en elle ni sujet regardant ni objet regardé, mais « un fait unique indépendant et auto-suffisant ${ }^{18}$ ", que Nishida mettra plus tard en lumière comme autoidentité absolument contradictoire.

La notion d'auto-identité absolument contradictoire (Zettai mujun teki jiko dôitsu) résume toute la logique de Nishida et constitue le fondement de sa philosophie. Élaborée entre 1925 et 1945, elle a permis à Nishida de remettre en question

both the perspective of ordinary logic, which seeks to eliminate paradox - i.e., which takes the law of non-contradiction as its emblem (either a thing is, or is not) - and the perspective of dialectic, which eliminates paradox and contradiction in a series of syntheses, and ultimately rests on a final synthesis of all into a single whole or oneness. What Nishida struggled for was a different perspective which could embrace both the thesis and the antithesis, the subject and the object, without suppressing either. The real, phenomenal world is both one and many, subject and objective, changing and

17 NiCHIDA Kitarô, Zen no kenkyû (Étude du bien) dans : Nishida Kitarô zenshû (Oeuures complètes de Nishida Kitarô, Tôkyô, Iwanami Shoten, 1965, 19 volumes), vol. 1, p. 9. À l'avenir: NKZ... Pour la traduction anglaise de cet ouvrage, voir NICHIDA Kitarô, A Study of Good. Tôkyô, Ministry of Education, 1960, 217 p.

18 Nakamura Yûjirô, "Nishida: le premier philosophe original au Japon", Critique 39/428-429 (1983) 32-54; p. 36. 
unchanging. Reality is self-contradictory ${ }^{19}$.

L'auto-identité absolument contradictoire tire son caractère opérationnel uniquement du fait que les termes opposés se situent eux-mêmes dans un lieu qui leur est commun. Comme le mentionne Nishida, "les choses qui s'opposent mutuellement doivent être extrêmement différentes d'un côté, et en même temps très proches de l'autre; elles doivent se situer dans un même genre ${ }^{20}$ \%. Cet universel le plus englobant au sein duquel les termes contradictoires sont originellement reliés et adviennent n'est autre que le néant absolu (zettai $m u$ ).

La logique paradoxale de Nishida présente le néant absolu comme la matrice logique de la détermination mutuelle des couples opposés. Il permet la néantisation d'un terme en auto-identité pour qu'apparaisse son contraire, et vice versa. Comprise à partir du point de vue du néant absolu, l'auto-identité absolument contradictoire de Nishida n'est rien d'autre que la reprise philosophique rigoureuse de la logique bouddhiste qui énonce que $A=A ; A=$ non- $A$; donc $A=A$. Le terme " auto-identité " (jiko dôitsu) correspond à " $A=A$ "; les mots " absolument contradictoire " (zettai mujun teki) correspondent à " $\mathrm{A}=$ non- $\mathrm{A}$ " (ou $\mathrm{A}$ n'est pas $\mathrm{A}$ mais devient $B$ ), c'est-à-dire à la néantisation de $A$ par l'intermédiaire du néant absolu. Cette néantisation est la condition même de l'auto-identité de $\mathrm{A}$ : "Le fait que le multiple se nie lui-même implique qu'il devient vraiment le multiple; le fait que l'un se nie lui-même implique qu'il devient vraiment l'un ${ }^{21}$ "; “Dans le monde de l'humain selon l'auto-identité contradictoire, le sujet doit être le sujet par le fait de disparaître dans l'environnement, et l'environnement doit être l'environnement par le fait de devenir sujet par auto-négation 22 ". Autrement dit, la clé de compréhension d'un terme est sa totale néantisation par l'intermédiaire du néant absolu, grâce à laquelle il renvoie à son contraire pour être ensuite réaffirmé.

Une exemplification très importante pour la philosophie moderne de ce processus de néantisation consiste dans les relations entre les indivi-

19 Robert E. CARTER, The Nothingness Beyond God, p. 62-63. Voir également NKZ 11, p. 398-399.

20 NisHidA Kitarô, Tetsugaku no konpon mondai (Problèmes fondamentaux de la philosophie), dans NKZ 7, p. 29. - Voir NisHIDA Kitarô, Fundamental Problems of Philosophy. Tôkyô, Sophia University, 1970, p. 14.

21 NiSHIDA Kitarô, "Rekishi teki sekai ni oite no kobutsu no tachiba " ("La situation de l'individuel dans le monde historique "), dans $N K 2$ 9, p. 77. Nishida élabore sa théorie des rapports inter-individuels principalement dans cet essai.

22 NiSHIDA Kitarô, "Zettai mujun teki jiko dôitsu " ("Auto-identité absolument contradictoire "), dans NKZ 9, p. 184. 
duels. Nishida présente la question par le paradoxe suivant : l'individuel (kobutsu) est ce qui existe et agit vraiment à partir de soi-même, et ce qui s'auto-détermine. Il est donc complètement indépendant et s'auto-détermine de l'intérieur. Mais en même temps, il n'y a pas d'individuel unique dans le monde réel (jitsuzai teki) où les choses agissent mutuellement; l'individuel n'est unique et isolé que dans le cas de la logique abstraite.

L'individuel qui s'auto-détermine de l'intérieur doit agir (kôi suru). Autrement, " il ne serait que la limite de la particularisation de l'universel $^{23}$ ". Le fait d'agir signifie déterminer (gentei suru) l'autre et être déterminé par lui. C'est ainsi que l'individuel se confronte (tai suru) vraiment à l'individuel. Ce qui détermine vraiment l'autre est ce qui se nie soi-même; agir est nier l'autre et êre nié par lui. Or, deux choses se transforment réciproquement lorsqu'elles agissent mutuellement. Le monde de la détermination mutuelle des individuels se constitue ici. Il peut sembler contradictoire que ce qui s'auto-détermine soi-même totalement de l'intérieur s'auto-détermine également totalement par le fait de se confronter à l'autre. Mais plus une chose est auto-contradictoire en ce sens, plus elle est individuelle. Nishida reprend ce paradoxe dans une formule qui revient constamment: "L'individuel est l'individuel par le fait de se confronter à l'individuel ${ }^{24}$ ».

Nishida considère que Leibniz avait entrevu que l'individuel est individuel par le fait de se confronter à l'individuel mais qu'il n'a pas examiné cette question de manière exhaustive, probablement à cause de l'intellectualisme de son époque. Il affirme même qu'on doit étudier la monadologie de Leibniz pour comprendre les relations entre les individuels. Lui-même fait de constantes références à Leibniz dans son œuvre. Il établit l'ensemble de sa théorie de l'individuel à partir de certains points précis de la monadologie, à savoir (1) la monade sans fenêtres, (2) l'agir réciproque des monades et (3) l'harmonie prétablie ${ }^{25}$. Il se sert de ces points pour dégager les thèmes les plus importants de sa propre problématique : (1) l'individuel, absolument indépendant (la monade sans fenêtres), (2) est en même temps l'individuel dans la mesure où il se confronte à l'individuel. (3) Ces relations entre individuels ne sont pas déterminées par une harmonie préétablie au sens leibnizien mais s'inscrivent dans le processus de l'auto-identité absolument contradictoire.

Les principales ressemblances et différences entre le système de

NiSHIDA Kitarô, " La situation de l'individuel dans le monde historique », p. 72.

NISHIDA Kitarô, " La situation de l'individuel dans le monde historique », p. 70.

25 Gottried Wilhelm LelBNiz, La monadologie. [Livre de poche, 4606], Paris, Grasset, 1991, 317 p. Voir, sur ces questions, les numéros 7, 49-52, 56-61. 
l'harmonie préétablie de Leibniz et la notion d'auto-identité absolument contradictoire de Nishida sont les suivantes. L'harmonie préétablie et l'auto-identité contradictoire servent toutes deux de réponse à la même question intiale, quoique formulée différemment par Leibniz et par Nishida : comment la monade leibnizienne, substance simple et ne comportant pas de fenêtres, c'est-à-dire ne recevant aucune influence de l'extérieur, peut-elle agir et entrer en relation avec l'infinité des autres monades qui constitue l'univers? Comment l'individuel nishidéen, absolument indépendant et auto-déterminé, peut-il être en même temps constamment et absolument en relation avec d'autres individuels?

On trouve, tant du côté de Leibniz que de celui de Nishida, un élément totalement simple et indépendant d'une part, et d'autre part, la question de la possibilité de l'agir mutuel et des relations de cet élément simple et indépendant (monade ou individuel) avec d'autres éléments simples et individuels. Leibniz explique cet agir mutuel (qu'il considère comme seulement apparent) et ces relations à l'aide de l'harmonie préétablie, laquelle consiste en des lois de développement immuables inscrites par Dieu dans chaque monade. Ce qui implique que le développement et l'agir de la monade sont établis à l'avance par Dieu et s'inscrivent entièrement dans un cadre précis auquel la monade doit se conformer et qui lui permet de se développer en harmonie avec l'ensemble des autres monades. Même la formation de composés semble être chez Leibniz de peu d'importance; ceux-ci sont de l'ordre du phénomène plutôt que de l'ordre d'une création nouvelle résultant de l'interaction véritable des monades, comme c'est le cas dans la théorie nishidéenne de l'individuel.

La notion d'auto-identité absolument contradictoire laisse supposer, contrairement au système de l'harmonie préétablie des monades, que tout dans le monde, et en particulier l'agir réciproque des individuels, est de l'ordre du processus, et que cet agir réciproque crée véritablement quelque chose de nouveau. On ne trouve pas dans l'individuel de lois préétablies inscrites par le divin et auxquelles il devrait se conformer au cours de son développement, c'est-à-dire de système initial de préformation tel que l'harmonie préétablie. Le développement et l'agir mutuel des individuels sont processuels, et pour ainsi dire spontanés. Autrement dit, ils n'ont pas leur raison d'être dans quelque chose d'extérieur à eux, en l'occurrence dans une harmonie préétablie par Dieu, mais en eux-mêmes.

Dans son essai intitulé "Vers une philosophie de la religion avec le concept d'harmonie préétablie comme guide ${ }^{26}$ ", Nishida met l'accent sur

26 NiSHIDA Kitarô, "Yotei Chôwa wo tehiki toshite shûkyô tetsugaku he " ( Vers une philosophie de la religion avec l'harmonie préétablie comme guide»), 
l'auto-identité absolument contradictoire (zettai mujun teki jiko dôitsu) impliquée dans un tel concept d'individuel puisque les individuels en confrontation mutuelle sont médiatisés par la négation absolue. Cette notion, qui implique une négation absolue sok $\mathrm{u}^{27}$ une affirmation absolue, est essentielle pour la compréhension du concept d'individuel.

Le néant absolu, basho (lieu) de l'interaction des individuels, instaure le processus de l'auto-identité contradictoire, au cours duquel les individuels agissent, c'est-à-dire se confrontent mutuellement par auto-négation. Ce processus qui se montre à travers le développement et l'agir mutuel des individuels peut être dégagé de la même manière dans tous les autres types de rapports qu'on trouve dans l'univers, à savoir les rapports entre l'individu et l'environnement (kojin to kankyô), entre le divin et le monde ( $k a m i$ to sekai), entre l'absolu et l'individu (zettaisha to kojin), et entre le je et le tu (watashi to nanji).

La relation entre individuels comporte, comme cas particulier, une notion fondamentale qui recoupe l'idée d'intersubjectivité des temps modernes : la relation je-tu (watashi to nanji) ${ }^{28}$. Les individuels (kobutsu) qui se confrontent mutuellement ne sont autres que ce je et ce tu. Cette relation est d'autant plus importante qu'elle permet de mettre d'avantage l'accent sur le basho du néant absolu et sur le processus de néantisation absolue qu'il suscite.

L'individu (kojin) ne naît pas à partir de lui-même mais est déterminé nécessairement à partir de l'environnement, tout comme les innombrables individus. Autrement dit, le je et le tu naissent à partir d'un même universel (ippansha) et se situent en lui en tant qu'auto-déterminations de cet universel; c'est ce qui leur vaut d'être en relation mutuelle et d'agir réciproquement comme personnalités. Le je ne s'instaure donc pas à partir de la conscience du soi individuel ( $k$ ojin teki) mais à partir de la société (shakai) qui doit, en un certain sens, être pensée avant la conscience

dans : NKZ 11, p. 114-146.

27 Le terme soku, qui est emprunté à la langue chinoise et qui est la traduction du rupam sunyata du Prajnaparamitra-sutra, indique l'identité paradoxale de la forma (rupa) et du vide (sunyata). En d'autres termes, l'affirmation et la négation sont en situation de tension dynamique.

28 La relation je-tu (watashi to nanji) de Nishida se rapproche beaucoup de la relation Ich-Du (ware to nanji) de Martin Buber. Nishida est en effet entré en contact avec la pensée de Buber sur cette question par l'intermédiaire de la théologie dialectique de Gogarten, entre autres. Voir NISHIDA Kitarô, "Watashi to nanji " (" Je et tu "), dans : NKZ 6, p. 341-427; Martin BUBER, Je et tu. Paris, Aubier Montaigne, 1970, 172 p. 
individuelle ${ }^{29}$.

La situation du je et du tu dans un même universel et leur détermination par ce dernier impliquent également leur médiatisation par la négation absolue (zettai hitei). Le lieu où le je et le tu se situent et qui entraîne l'auto-négation de chacun d'entre eux, c'est-à-dire le basho du néant absolu, est l'intermédiaire où s'unit ce qui ne s'unit absolument pas, l'intermédiaire où le caractère absolu de chaque élément et l'aspect d'absolue confrontation s'unissent dynamiquement. Pareille négation ouvre dans le basho du néant absolu un intervalle insondable qui est la condition même de la subjectivité (shutaisei) ${ }^{30}$ du je et du tu. C'est là une manière de se joindre en se coupant, c'est-à-dire en ne se touchant pas directement. Cela s'associe directement au fait que le soi est le soi sans être le soi, c'est-à-dire comporte une interruption en lui-même. Nishida mentionne que « le je est le je par le fait de reconnaître la personnalité du tu, et le tu est le tu par le fait de reconnaître la personnalité du je. Ce qui fait du tu le tu est le je, et ce qui fait du je le je est le tu. Le je et le tu étant une discontinuité absolue, le je détermine le tu et le tu détermine le $\mathrm{je}^{31} »$.

La négation absolue, qui est unification négatrice, union d'éléments absolument détachés et continuité de la discontinuité, est introduite dès le début au cœur de la relation je-tu. Là, l'auto-identité de ce qui est absolument contraire est pensée à partir de l'expérience faite par le soi personnel que le tu est le tu par le fait de se confronter au je, et que le je est le je par le fait de se confronter au tu.

\section{WATsuji Tetsurô. Logique de la relationalité32}

La philosophie de Watsuji se fonde en partie sur celle de Nishida. Tant Nishida que Watsuji ont mis l'accent sur l'idée orientale énonçant que seule une expérience de négation permet l'expérience de la réalité et son affirmation. On remarque l'influence de Nishida lorsque Watsuji réfère à la négation absolue.

29 Watsuji a exactement ce même souci de déconstruction de la subjectivité des temps modernes en vue de montrer les liens entre individuel et société.

30 La langue japonaise a deux mots pour traduire la subjectivité. Le premier, shukansei, s'oppose à l'objectivité (kyakkansei) et désigne de niveau du théorique, du cognitif, de la perception; c'est le sujet qui voit. Le second, shutaisei, désigne le sujet agissant. C'est de ce dernier dont il est ici question.

31 NishidA Kitarô, "Je et tu ", p. 415.

Voir le « Glossaire ". 
La pensée de Watsuji se divise en trois périodes. Durant la première période (1913-1919), Watsuji met l'accent sur la « volonté de pouvoir » de Nietzsche et sur l'esprit d' "élite", ainsi que sur la renaissance poétique du culte de la nature qu'on trouve dans la Grèce ancienne. La deuxième période (1920-1930) est celle des études historiques (bouddhisme et christianisme). La troisième période (1932-1952) est la plus importante puisque c'est à ce moment que Watsuji établit son éthique, suite à ses études philosophiques et culturo-historiques. Les deux ouvrages majeurs de cette période sont Ethique (1937, 1942 et 1949) ${ }^{33}$ et L'éthique comme science de l'humain (1934) ${ }^{34}$.

Le premier volume de Éthique est celui où Watsuji traite le plus du néant absolu; il fait état des tendances orientales de l'éthique de Watsuji puisque celui-ci y met l'accent sur l'aspect négatif de la moralité : «Moral laws are originated through the absolute negation (zettai teki hiteisei) of the subject. The individual only through the negation of the self can join the whole of the nation. This absolute negation ends in the $k \hat{u}$ (the emptiness of the self), in order to reach the 'absolute totality', not dissimilar in purpose to Nishida's nothingness ${ }^{35}$ ». Watsuji montre, en s'aidant d'une analyse des sociologues Simmel et Durkheim entre autres, que la réalité sociale absorbe nettement l'individu. Ainsi, "man must dissolve himself in the quest for the absolute emptiness [...] because of the fundamental void intrinsic to man and society. Society, formed as it is of individuals, is but a dialectical development of self-denying units toward the great void which is social totality ${ }^{36}$ ". On remarque ici l'influence de Nishida et du bouddhisme.

Le premier chapitre de L'éthique comme science de l'humain contient les concepts éthiques fondamentaux de Watsuji; c'est là le texte principal

33 W ATSUJl Tetsurô, Rinrigaku ue, Rinrigaku shita (Éthique, première partie, Éthique, deuxième partie), dans : Watsuji Tetsurô zenshû (Euvres complètes de Watsuji Tetsurô, Tôkyô, Iwanami shoten, 1963, 20 volumes), vol. 10 et 11 . À l'avenir: WTZ...

34 W ATSUJI Tetsurô, Ningen no gaku toshite no rinrigaku (L'éthique comme science de l'humain). Tôkyô, Iwanami Shoten, 1989, 262 p. Une traduction du premier chapitre de cet ouvrage est disponible en anglais : WATSUJı Tetsurô, "The Significance of Ethics as the Study of Man ", Monumenta Nipponica 26/3-4 (1971) 395-413.

35 Gino PIOVESANA, Recent Japanese Philosophical Thought, 1862-1962 : a Survey. Tôkyô, Enderle Bookstore, 1968, 296 p.; p. 142.

36 Gino PIOvesana, Recent Japanese Philosophical Thought, p. 142-143. Le second volume de Éthique traite des problèmes éthiques de la famille, de la nation, des implications du "relationisme social ", ainsi que des communautés géographiques et culturelles et de leur éthos éthico-économique. Le troisième volume reprend les questions de la dimension historique de l'humain et du fûdo (climat). 
concernant les rapports entre néant absolu et relationalité. Le second chapitre montre comment Watsuji établit les principes du comportement éthique. Il considère que l'éthique, seule anthropologie possible, " consisted essentially in the aidagara or the relationship aspect of contacts between man and man, man and his family, man and society. Taken in its entirety, man is not only an individual or a mere social entity but a being essentially related to the social world to which he belongs ${ }^{37}$ ". Watsuji appelle cette éthique "science de l'humain", qu'il mène à l'aide de l'analyse des termes "éthique " (rinri), "humain " (ningen), "être-dans-le-monde" (yo no naka) et "existence" (sonzai). Ne pas prendre en compte le concept d'aidagara, qui a autant d'importance que les notions de néant absolu et de basho, empêche de se rendre compte de la relation éthique qui relie intrinsèquement les humains.

Watsuji s'efforce, tout comme Nishida, de remettre en question la conception individualiste de l'être humain qu'a la modernité. Un acquis important dans l'histoire de la philosophie est que l'individu est l'achèvement de l'esprit moderne. C'est toutefois la raison pour laquelle l'éthique est conçue seulement comme le problème de la conscience individuelle. Watsuji soutient que

this view of isolated subjectivity which abstracts from the behavioral relationships between men is then erroneously applied even to ethical questions. The results is that the area of ethical questions also becomes limited to the relationship between human subjectivity and nature, within which relation the sphere of the self is handled as a problem of the will in contrast to the problem of cognition ${ }^{38}$.

De cette façon, on place au centre de l'éthique des questions telles que l'indépendance du soi face à la nature, l'autonomie du soi, et l'autosatisfaction des désirs. L'anthropologie et la psychologie spéculatives allemandes envisagent l'humain de manière abstraite en traitant de dichotomies telles que l'âme et le corps, l'esprit et la matière. L'humain y est abstrait de son groupe social et y est considéré comme une chose existant par elle-même. De ce fait, la question de l'humain est réduite à l'unité du corps et de l'esprit ou bien à la question du soi et de l'individu. D'ailleurs, les mots occidentaux "anthropos", "homo", "man", et "Mensch" désignent d'abord l'individu humain. C'est pourquoi on ne devrait pas pouvoir les utiliser pour rendre compte de la relationalité entre humains, de

Piovesana, Recent Japanese Philosophical Thought, p. 141. L'éthique comme science de l'humain présente également des analyses des systèmes éthiques d'Aristote, de Kant, de Cohen, de Hegel, de Feuerbach et de Marx.

38 W ATSujl, "The Significance of Ethics as the Study of Man », p. 395-396. 
l'existence commune et de la société. Les questions éthiques ne peuvent se résumer à la notion d'individu, qui n'est qu'une partie de l'existence humaine et ne peut être substituée à l'humain total. Elles se situent plutôt dans la "relationalité entre humains". Watsuji affirme que "a man must be able to exist individually and at the same time must be a social being ${ }^{39}$ ». Dans ce contexte, l'anthropologie et les sciences sociales ne peuvent plus être considérées comme deux sciences séparées l'une de l'autre et, conséquemment, abstraites de l'humain concret.

En mettant l'accent sur le fait que l'individualisme moderne est basé sur une compréhension de l'individu coupé de la société, Watsuji aboutit au problème fondamental de la nature de l'espace et du temps ${ }^{40}$. Heidegger, dont Watsuji fut le contemporain, aurait lui aussi de l'humain une telle vision partielle. La lecture que Watsuji fit de Sein und Zeit lui montra que Heidegger met l'accent surtout sur la temporalité du Dasein, comprise comme mode d'être fondamental de l'humain. Le temps est plus fondamental que l'espace car le mode selon lequel le Dasein est dans le monde (In-der-Welt-Sein) est celui d'une prise subjective sur le temps, qui saisit le passé et le futur. De ce fait, la perspective de Heidegger risque de faire perdre de vue l'importance pourtant décisive de la spatialité. Il semble que la limite de son œuvre soit de n'avoir pas mis en lumière la structure fondamentale du Dasein comme spatialité.

Comme c'est bien connu, Heidegger insiste sur la temporalité lorsqu'il traite du Sein-zum-Tode (être-vers-la-mort) dans la deuxième partie de Sein und Zeit. Or, c'est la trop grande emphase mise sur l'individualité qui entraîne Heidegger à mettre l'accent sur la catégorie de temporalité au détriment de celle de spatialité, selon Watsuji. Ce dernier mentionne, dans la préface de Fûdo (Le climat), que " le Dasein était le Dasein de l'individuel seulement ${ }^{41}$ ". La temporalité étant liée à l'aspect subjectif de l'existence, la spatialité est objectivée simplement à titre de nature ou d'environnement. Watsuji soutient que toute la philosophie se trouve biaisée lorsqu'on écarte d'emblée la spatialité et l'aspect social de l'humain pour mettre l'accent seulement sur la temporalité et l'individualité.

Or, la vraie temporalité devrait toujours être envisagée en lien avec

W ATSUJI, « The Significance of Ethics as the Study of Man », p. 399.

40 À propos de la spatio-temporalité chez Watsuji, voir WATsujı Tetsurô, “Ningen sonzai no kûkanteki-jikanteki kôzô " ( Structure spatiale et temporelle de l'existence humaine "), dans: WTZ 10, p. 152-329.

41 Watsujı Tetsurô, Fâdo (Le climat), Tôkyô, Iwanami shoten, 1967. Une traduction de cet ouvrage est disponible en anglais : W ATSUjI Tetsurô, A Climate. A Philosophical Study, Tôkyô, Japanese National Commission for UNESCO, 1961, 235 p. 
la spatialité. C'est ce que fait Watsuji en mettant l'accent autant sur la spatialité et l'aspect social de l'existence humaine que sur la temporalité et l'aspect individuel de cette même existence humaine. Heidegger aurait pu franchir ce pas s'il avait considéré le dasein comme ningen (humain en relation), et non pas seulement comme hito (personne individuelle). Autrement dit, le Dasein devrait être envisagé sous son double aspect individuel et social. La prise en compte de cette double structure est la condition de la mise en relation de la temporalité (accent mis sur l'individuel) et de la spatialité (accent mis sur le social). Nous existons dans un basho spatial défini qui est le lieu des interconnections du monde vivant.

La double nature de l'humain, à savoir son individualité et sa sociabilité, s'exprime fort bien dans le mot japonais ningen (humain), qui ne désigne ni seulement les humains, ni seulement la société :

As long as ningen are men, men as individuals always will differ from sociery as a whole. Because they are not society as a whole, they are individual men. And hence one individual is not entirely the same as another individual; the self and the other are absolutely "other " to each other. And yet as long as men (ningen) are society (yo no naka), there is always a communal situation; there is society and not merely isolated individuals. Precisely because there are not isolated individuals there is man (ningen). Consequently, self and other, though mutually they are absolutely "other ", are nevertheless one in communal existence. Individuals, who fundamentally differ from society as a whole, are yet immersed within society. Man (ningen) is this kind of relational unity of opposites. The essence of man cannot be understood without observing this dialectical structure ${ }^{42}$.

La double nature de l'humain (ningen) est un mouvement de négation qui s'explique de la manière suivante : un individu indépendant et autosuffisant - et Nishida l'avait déjà affirmé avec force - n'est que pure abstraction. Il doit comporter un caractère de négation par lequel il nie l'ensemble de l'humanité et grâce auquel il se constitue véritablement comme individu et, dans le même mouvement, comme société. L'inverse est également vrai. La société se constitue par la négation de l'individualité. Lorsque l'individu est nié, il nie aussitôt la totalité à son tour pour devenir un individu. Ce mouvement de négation permet au tout d'exister. De cette manière, la double nature de l'humain (individu et société, hito to yo no naka) se présente comme un mouvement unique impliquant une double négation ou une négation de la négation, comprise comme 
the self-returning, realizing movement of the absolute whole, and it is truly human relationship. Therefore the fundamental principle of human relations must be nothing else but realization through individuals (i.e. negations of the totality) of their totality (i.e. negation of the negation). It is ultimately the movement of self-realization of an original, absolute totality ${ }^{43}$.

Il y a donc deux mouvements dans le principe fondamental des relations humaines. Le premier est l'individuel qui se situe comme autre devant le tout. C'est là la première étape de la conscience de soi puisque sans individuel, il n'y aurait pas de relations humaines. Le second mouvement est l'absorption de l'individuel dans le tout. Cette absorption dans le tout est également la condition des relations humaines. Ce qui ne veut pas dire que l'individualité disparaît purement et simplement puisque sitôt absorbé, l'individuel répète le mouvement de négation en niant à son tour la totalité.

On peut donc qualifier l'esthétisme des japonais(es) de culture de l'aidagara ou relationalité axée sur une structure paradoxale de négation du sujet et de l'objet, ou encore, sur une affirmation absolue à travers une négation mutuelle. Le terme aidagara réfère à une immédiateté paradoxale qui précède la séparation entre le sujet et l'objet, et entre le soi et la société. Cela se rapproche beaucoup de ce que Nishida appelle "relation je-tu» et de ce que Tanabe appelle "logique de l'espèce 44 ». L'intersubjectivité éthique de Watsuji met l'accent sur un horizon expérientiel dans lequel le plan des individus abstraits est transcendé. Et l'humain et l'éthique sont fondés dans cet aidagara.

Watsuji soutient que sa structure d'aidagara permet de critiquer le manque d'un concept dont Aristote, Kant, Cohen, Hegel, Feuerbach et Marx auraient pu se servir pour désigner les relations dialectiques. Après avoir élaboré le thème de l'aidagara comme pendant japonais de l'analytique heideggérienne du Dasein, Watsuji l'a retracé dans différentes formes de shinto, de bouddhisme et de confucianisme au Japon. Dans son éthique, l'absolue négativité du sujet reprend l'éthique interrelationnelle du confucianisme.

43 W ATSUJl, "The Significance of Ethics as the Study of Man », p. 409.

44 Voir TANABE Hajime, “The Logic of The Species As Dialectics " : Monumenta Nipponica 24 (1969 - no 3) 273-288. 


\section{Conclusion}

L'orientation fondamentale de la pensée japonaise traditionnelle et de la philosophie japonaise contemporaine est le néant absolu, centre vide vers lequel converge toute la réalité, qui permet tous les types de relations et suscite tous les types de discours, sans jamais être lui-même nommé ou thématisé. Les philosophies de Nishida et de Watsuji convergent vers ce centre vide, s'articulent autour de ce centre vide, en même temps qu'elles sont englobées par lui.

\section{Glossaire}

NiSHITANI Keiji (1900-1990) a étudié avec Nishida dans les années '20, puis en Allemagne avec Martin Heidegger, avant la deuxième guerre mondiale. De retour au Japon, il a enseigné l'allemand et l'éthique à Kyôto, de 1928 à 1935 et, de 1935 à 1955, la religion. De 1955 à 1963, il a occupé la chaire de philosophie moderne de l'Université de Kyôto. Nishitani est connu en Occident surtout par six de ses essais qui ont été publiés en anglais sous le titre Religion and Nothingness (Berkeley and Los Angeles, University of California Press, 1982, 317 p.) et, plus récemment, par son ouvrage intitulé The Self-Overcoming of Nihilism (Albany, State University of New York Press, 1990, 240 p.). Ces ouvrages poursuivent l'effort engagé par NISHIDA Kitarô de rendre compte de l'héritage oriental à partir de la philosophie et de la science occidentales. Nishitani est considéré comme l'héritier de Nishida.

La carrière de TANABE Hajime se développa d'abord indépendamment de celle de Nishida. Après avoir gradué au Département de philosophie de l'Université de Tôkyô en 1908, il devint, en 1913, lecteur de philosophie des sciences à l'Université Tôhoku de Sendai. En 1919, il commença à enseigner à l'Université de Kyôto, où il devint le successeur de Nishida le plus célèbre.

L'École de Kyôto naquit vers 1920, avec les œuvres de NISHIDA Kitarô. La philosophie de Nishida attira rapidement des disciples, bien que Nishida n'ait pas eu lui-même l'intention de fonder une école. Les principaux disciples de Nishida sont les suivants : TANABE Hajime (18851962), HISAMATSU Shinichi (1889-1981), NISHITANI Keiji (1900-1990), TAKEUCHI Yoshinori (né en 1913), TSUJIMURA Kôichi (né en 1922) et UEDA Shizuteru (né en 1926). L'École de Kyôto a son siège à la chaire de philosophie de la religion de l'Université de Kyôto. Les philosophes à occuper cette chaire furent, successivement, les suivants: NISHIDA Kitarô, TANABE Hajime, TAKEUCHI Yoshinori, NISHITANI Keiji, UEDA Shizuteru et HASE Shôtô. La majorité des philosophes japonais contemporains, dont les plus éminents sont Tanabe, Nishitani et Watsuji, élaborè- 
rent et précisèrent leurs concepts philosophiques en confrontation avec la philosophie de Nishida. Leur thème central n'est autre que le néant absolu.

SUZUKI Daisetsu Teitarô est né en 1870 à Kanazawa. C'est à la Quatrième École supérieure de cette ville qu'il connut Nishida, qui demeura toute sa vie son ami intime. Il séjourna aux États-Unis de 1897 à 1909, et en Europe en 1910. Il épousa l'américaine Beatrice Lane en 1911 lors de son retour au Japon. C'est avec elle qu'il édita la revue Eastern Buddhist. Après avoir été professeur d'anglais, il devint professeur à l'Université d'Otani de Kyôto. Il séjourna de nouveau aux États-Unis et en Europe de 1951 à 1958 et mourut à Kamakura (tout comme Nishida) en 1966, à l'âge de 96 ans. Il s'intéressa au bouddhisme japonais, notamment à la secte zen et à la Vraie secte de la Terre pure (jodoshinshu), qu'il a situées dans le cadre de la culture japonaise. Il fut un représentant du dialogue entre les cultures occidentales et la culture japonaise puisqu'il écrivit la moitié de son ceuvre en anglais. Un des résultats de son travail fut la diffusion de la connaissance du bouddhisme zen en Occident. Grâce à lui, les occidentaux se sont rendus compte de l'importance de comprendre le bouddhisme pour accéder à la culture japonaise.

NISHIDA Kitarô (1870-1945) naquit à Unoge en 1870. Il devint professeur de lycée dans la ville de Kanazawa après avoir terminé ses études à la Faculté des lettres de l'Université de Tôkyô en 1894. En 1898, il fut nommé à ce qui est aujourd'hui l'Université de Kanazawa, où il enseigna durant dix ans. Il fut nommé professeur adjoint à la Faculté des lettres de l'Université de Kyôto en 1910, où il enseigna l'éthique et la science des religions. Il devint professeur titulaire de la première chaire de philosophie de l'Université de Kyôto trois ans plus tard, après avoir reçu son grade de doctorat. C'est là qu'il enseigna jusqu'à sa retraite, en 1928. Il mourut en 1945 à Kamakura. Il est considéré comme le philosophe japonais le plus important.

WATSUjI Tetsurô naquit en 1889 à Himeji, dans la préfecture de Hyôgo, et mourut en 1960. Il entra du Département de philosophie de l'Université de Tôkyô en 1909 après avoir hésité entre la philosophie et la littérature anglaise. On remarque des talents littéraires dans son œuvre; il est même considéré comme un écrivain de l'époque Shôwa. D'ailleurs, on dit qu'il fut influencé, au cours de sa jeunesse, par les dernières nouvelles de NATSUME Sôseki. Il étudia en Allemagne en 1927 et 1928 puis devint, en 1931, professeur à l'Université de Kyôto, puis à l'Université de Tôkyô en 1934. Il prit sa retraite en 1949. Watsuji ne fait pas partie de l'École de Kyôto. La raison principale en est qu'il fit carrière à l'Université de Tôkyô plutôt qu'à l'Université de Kyôto. Pourtant, la parenté entre sa philosophie et celle de Nishida n'en est pas moins frap- 
pante. Watsuji est connu parmi les philosophes japonais contemporains comme un éthicien, un historien de l'éthique japonaise, et un historien des idées et de la culture, tant orientale qu'occidentale. Il a fait en éthique ce que Nishida a accompli en philosophie, à savoir exprimé les problèmes de la pensée orientale dans les catégories occidentales. Watsuji est plus systématique que Nishida problablement parce qu'il s'est limité au domaine de l'éthique (Nishida n'a pas construit de philosophie systématique, tâche qui incombe à ses disciples à partir de ses écrits). Les principaux thèmes de son système reviennent dans chacune de ses ouvres. 